\title{
Optimal Bounds for Gaussian Arithmetic-Geometric Mean with Applications to Complete Elliptic Integral
}

\author{
Hua Wang, ${ }^{1,2}$ Wei-Mao Qian, ${ }^{3}$ and Yu-Ming $\mathrm{Chu}^{4}$ \\ ${ }^{1}$ Department of Mathematics and System Science, National University of Defense Technology, Changsha 410073, China \\ ${ }^{2}$ Department of Mathematics, Changsha University of Science and Technology, Changsha 410014, China \\ ${ }^{3}$ School of Distance Education, Huzhou Broadcast and TV University, Huzhou 313000, China \\ ${ }^{4}$ School of Mathematics and Computation Sciences, Hunan City University, Yiyang 413000, China
}

Correspondence should be addressed to Yu-Ming Chu; chuyuming2005@126.com

Received 8 May 2016; Revised 11 June 2016; Accepted 23 June 2016

Academic Editor: Rudolf L. Stens

Copyright (c) 2016 Hua Wang et al. This is an open access article distributed under the Creative Commons Attribution License, which permits unrestricted use, distribution, and reproduction in any medium, provided the original work is properly cited.

We present the best possible parameters $\alpha_{1}, \beta_{1}, \alpha_{2}, \beta_{2} \in \mathbb{R}$ and $\alpha_{3}, \beta_{3} \in(1 / 2,1)$ such that the double inequalities $Q^{\alpha_{1}}(a, b) A^{1-\alpha_{1}}(a$, b) $<A G[A(a, b), Q(a, b)]<Q^{\beta_{1}}(a, b) A^{1-\beta_{1}}(a, b), \alpha_{2} Q(a, b)+\left(1-\alpha_{2}\right) A(a, b)<A G[A(a, b), Q(a, b)]<\beta_{2} Q(a, b)+\left(1-\beta_{2}\right) A(a, b)$, $Q\left[\alpha_{3} a+\left(1-\alpha_{3}\right) b, \alpha_{3} b+\left(1-\alpha_{3}\right) a\right]<A G[A(a, b), Q(a, b)]<Q\left[\beta_{3} a+\left(1-\beta_{3}\right) b, \beta_{3} b+\left(1-\beta_{3}\right) a\right]$ hold for all $a, b>0$ with $a \neq b$, where $A(a, b), Q(a, b)$, and $A G(a, b)$ are the arithmetic, quadratic, and Gauss arithmetic-geometric means of $a$ and $b$, respectively. As applications, we find several new bounds for the complete elliptic integrals of the first and second kind.

\section{Introduction}

Let $r \in(0,1)$ and $a, b>0$. Then the elliptic elliptic integral of the first kind $\mathscr{K}(r)$ and second kind $\mathscr{E}(r)$, Gaussian arithmetic-geometric mean $A G(a, b)$, arithmetic mean $A(a, b)$, and quadratic mean $Q(a, b)$ are, respectively, given by

$$
\begin{aligned}
\mathscr{K}(r) & =\int_{0}^{\pi / 2} \frac{1}{\sqrt{1-r^{2} \sin ^{2} t}} d t, \\
\mathscr{E}(r) & =\int_{0}^{\pi / 2} \sqrt{1-r^{2} \sin ^{2} t} d t, \\
A G(a, b) & =\frac{\pi}{2 \int_{0}^{\pi / 2}\left(d t / \sqrt{a^{2} \cos ^{2} t+b^{2} \sin ^{2} t}\right)}, \\
A(a, b) & =\frac{a+b}{2}, \\
Q(a, b) & =\sqrt{\frac{a^{2}+b^{2}}{2}} .
\end{aligned}
$$

The Gauss identity [1-3] shows that

$$
A G\left(1, r^{\prime}\right)=\frac{\pi}{2 \mathscr{K}(r)}
$$

for all $r \in(0,1)$, where and in what follows $r^{\prime}=\sqrt{1-r^{2}}$.

It is well known that the elliptic elliptic integrals $\mathscr{K}(r)$ and $\mathscr{E}(r)$ and the Gaussian arithmetic-geometric mean $A G(a, b)$ have many applications in mathematics, physics, mechanics, and engineering [4-9]. Recently, the bounds for the Gaussian arithmetic-geometric mean $A G(a, b)$ have attracted the attention of many researchers.

The inequalities

$$
\begin{aligned}
\frac{1+\sqrt{r}}{2} A G(1, \sqrt{r}) & <A G(1, r)<\frac{\pi}{2 \log (4 / r)}, \\
L(a, b) & <A G(a, b)<L_{3 / 2}(a, b)
\end{aligned}
$$

for all $r \in(0,1)$ and $a, b>0$ with $a \neq b$ can be found in the literature [10-12], where $L(a, b)=(a-b) /(\log a-\log b)$ and $L_{p}(a, b)=L^{1 / p}\left(a^{p}, b^{p}\right)$ are, respectively, the logarithmic and $p$ th generalized logarithmic means of $a$ and $b$. The first inequality of (5) is due to Carlson and Vuorinen [13]. 
By using a variant of L'Hospital's rule and representation theorems with elliptic integrals, Vamanamurthy and Vuorinen [14] proved, among other results, the inequalities

$$
\begin{aligned}
A G(a, b) & <\sqrt{A(a, b) L(a, b)}, \\
L(a, b) & <A G(a, b)<\frac{\pi}{2} L(a, b), \\
A G(a, b) & <I(a, b)<A(a, b), \\
A G(a, b) & <\frac{A(a, b)+G(a, b)}{2}, \\
A(a, b) & <\frac{A G\left(a^{2}, b^{2}\right)}{A G(a, b)}<Q(a, b), \\
A G(a, b) & >L^{1 / \lambda}\left(a^{\lambda}, b^{\lambda}\right)
\end{aligned}
$$

for all $a, b>0$ with $a \neq b$ and $\lambda \in(0,1]$, where $I(a, b)=$ $\left(b^{b} / a^{a}\right)^{1 /(b-a)} / e$ is the identric mean of $a$ and $b$.

By use of the homogeneity of the above means and a series representation of $A G(a, b)$ due to Gauss, Sándor [15] obtained, among other results, new proofs for inequalities (7), (8) and a counterpart of inequality (9):

$$
A G(a, b)>\sqrt{A(a, b) G(a, b)},
$$

for all $a, b>0$ with $a \neq b$, where $G(a, b)=\sqrt{a b}$ is the geometric mean of $a$ and $b$. Inequalities (9) and (12) show that $A G$ lies between the arithmetic and geometric means of $A$ and G. In [16], Sándor provided new proofs for inequalities (6) and (8), (9), (10), and (12) by using only elementary methods for recurrent sequences and found much stronger forms of these results.

Neuman and Sándor [17] gave the comparison of the Gaussian arithmetic-geometric mean and the Schwab-Borchardt mean.

The upper bounds $\pi /[2 \log (4 / r)]$ for $A G(1, r)$ in (4) were replaced by $\pi\left(1-r^{2} / 9\right) /[2 \log (4 / r)]$ due to Kühnau [18].

Qiu and Vamanamurthy [19] presented that $4 \pi /[(9-$ $\left.\left.r^{2}\right)(2 \log 2-\log r)\right]$ and $\left(9-r^{2}\right) \pi /[18.192 \times(2 \log 2-\log r)]$ are, respectively, the lower and upper bounds for $A G(1, r)$ with $r \in(0,1)$. Alzer and Qiu [20] proved that $\lambda=3 / 4$ and $\mu=2 / \pi$ are the best possible parameters such that the double inequality

$$
\begin{gathered}
\frac{1}{\lambda / L(a, b)+(1-\lambda) / A(a, b)}<A G(a, b) \\
<\frac{1}{\mu / L(a, b)+(1-\mu) / A(a, b)}
\end{gathered}
$$

holds for all $a, b>0$ with $a \neq b$.

Chu and Wang [21] proved that the double inequality

$$
S_{p}(a, b)<A G(a, b)<S_{q}(a, b)
$$

holds for all $a, b>0$ with $a \neq b$ if and only if $p \leq 1 / 2$ and $q \geq 1$, where $S_{p}(a, b)=\left[\left(a^{p-1}+b^{p-1}\right) /(a+b)\right]^{1 /(p-2)}(p \neq 2)$ and $S_{2}(a, b)=\left(a^{a} b^{b}\right)^{1 /(a+b)}$ is the $p$ th Gini mean of $a$ and $b$. In [22], Yang et al. proved that the inequalities

$$
\begin{gathered}
S_{7 / 4,-1 / 4}(a, b)<A G(a, b)<A^{1 / 4}(a, b) L^{3 / 4}(a, b), \\
A G(a, b)<\sqrt{S_{p, 1}(a, b) S_{1-p, 1}(a, b)}
\end{gathered}
$$

hold for all $p \in(1 / 2,1)$ and $a, b>0$ with $a \neq b$, where $S_{p, q}(a, b)=\left[q\left(a^{p}-b^{p}\right) /\left(p\left(a^{q}-b^{q}\right)\right)\right]^{1 /(p-q)}$ is the Stolarsky mean [23] of $a$ and $b$.

Let $a, b>0$ with $a \neq b$ and $x \in[1 / 2,1]$. Then it is not difficult to verify that the function $f(x)=Q[x a+(1-x) b, x b+$ $(1-x) a]$ is continuous and strictly increasing on the interval $[1 / 2,1]$. Note that

$$
\begin{aligned}
& f\left(\frac{1}{2}\right)=A(a, b)=\min \{A(a, b), Q(a, b)\} \\
& <A G[A(a, b), Q(a, b)], \\
& A G[A(a, b), Q(a, b)]<\max \{A(a, b), Q(a, b)\} \\
& =Q(a, b)=f(1) \text {. }
\end{aligned}
$$

Inequalities (16) give us the motivation to deal with the best possible parameters $\alpha_{1}, \beta_{1}, \alpha_{2}, \beta_{2} \in \mathbb{R}$ and $\alpha_{3}, \beta_{3} \in$ $(1 / 2,1)$ such that the double inequalities

$$
\begin{aligned}
& Q^{\alpha_{1}}(a, b) A^{1-\alpha_{1}}(a, b)<A G[A(a, b), Q(a, b)] \\
& \quad<Q^{\beta_{1}}(a, b) A^{1-\beta_{1}}(a, b), \\
& \alpha_{2} Q(a, b)+\left(1-\alpha_{2}\right) A(a, b)<A G[A(a, b), Q(a, b)] \\
& \quad<\beta_{2} Q(a, b)+\left(1-\beta_{2}\right) A(a, b), \\
& Q\left[\alpha_{3} a+\left(1-\alpha_{3}\right) b, \alpha_{3} b+\left(1-\alpha_{3}\right) a\right] \\
& \quad<A G[A(a, b), Q(a, b)] \\
& \quad<Q\left[\beta_{3} a+\left(1-\beta_{3}\right) b, \beta_{3} b+\left(1-\beta_{3}\right) a\right]
\end{aligned}
$$

hold for all $a, b>0$ with $a \neq b$.

\section{Lemmas}

In order to prove our main results we need several derivative formulas and particular values for $\mathscr{K}(r)$ and $\mathscr{E}(r)$, which we present in this section.

$\mathscr{K}(r)$ and $\mathscr{E}(r)$ satisfy the formulas (see [24])

$$
\begin{aligned}
& \frac{d \mathscr{K}(r)}{d r}=\frac{\mathscr{E}(r)-r^{\prime 2} \mathscr{K}(r)}{r r^{\prime 2}}, \\
& \frac{d \mathscr{E}(r)}{d r}=\frac{\mathscr{E}(r)-\mathscr{K}(r)}{r}, \\
& \mathscr{K}\left(0^{+}\right)=\mathscr{E}\left(0^{+}\right)=\frac{\pi}{2}, \\
& \mathscr{K}\left(1^{-}\right)=\infty, \\
& \mathscr{E}\left(1^{-}\right)=1,
\end{aligned}
$$




$$
\begin{aligned}
& \mathscr{K}\left(\frac{\sqrt{2}}{2}\right)=\frac{\Gamma^{2}(1 / 4)}{4 \sqrt{\pi}}=1.85407467 \ldots, \\
& \mathscr{E}\left(\frac{\sqrt{2}}{2}\right)=\frac{4 \Gamma^{2}(3 / 4)+\Gamma^{2}(1 / 4)}{8 \sqrt{\pi}}=1.35064388 \ldots,
\end{aligned}
$$

where $\Gamma(x)=\int_{0}^{\infty} t^{x-1} e^{-t} d t(x>0)$ is the classical Euler gamma function.

Lemma 1 (see [24, Theorem 1.25]). Let $-\infty<a<b<\infty$, $f, g:[a, b] \rightarrow \mathbb{R}$ be continuous on $[a, b]$ and differentiable on $(a, b)$ and $g^{\prime}(x) \neq 0$ on $(a, b)$. Then both functions

$$
\begin{aligned}
& \frac{f(x)-f(a)}{g(x)-g(a)}, \\
& \frac{f(x)-f(b)}{g(x)-g(b)}
\end{aligned}
$$

are increasing (decreasing) on $(a, b)$ if $f^{\prime}(x) / g^{\prime}(x)$ is increasing (decreasing) on $(a, b)$. If $f^{\prime}(x) / g^{\prime}(x)$ is strictly monotone, then the monotonicity in the conclusion is also strict.

Lemma 2 (see [24, Theorem 3.21(1), Theorem 3.21(7), and Exercises 3.43(32)]). The following statements are true:

(1) The function $r \rightarrow\left[\mathscr{E}(r)-r^{\prime 2} \mathscr{K}(r)\right] / r^{2}$ is strictly increasing from $(0,1)$ onto $(\pi / 4,1)$.

(2) The function $r \rightarrow r^{\prime \lambda} \mathscr{K}(r)$ is strictly decreasing from $(0,1)$ onto $(0, \pi / 2)$ if $\lambda \geq 1 / 2$.

(3) The function $r \rightarrow[\mathscr{K}(r)-\mathscr{E}(r)] /\left[r^{2} \mathscr{K}(r)\right]$ is strictly increasing from $(0,1)$ onto $(1 / 2,1)$.

Lemma 3. Let $p=1 / 2+\sqrt{2} \sqrt{\pi^{2}-2 \mathscr{K}^{2}(\sqrt{2} / 2)} /[4 \mathscr{K}(\sqrt{2} /$ $2)]=0.8299 \ldots$ and $h(r)$ be defined by

$$
h(r)=\frac{\mathscr{E}(r)-r^{\prime 2} \mathscr{K}(r)}{r^{2} r^{\prime 2} \mathscr{K}^{3}(r)}-\frac{16 p(1-p)}{\pi^{2}} .
$$

Then there exists $r_{0} \in(0, \sqrt{2} / 2)$ such that $h(r)<0$ for $r \in$ $\left(0, r_{0}\right)$ and $h(r)>0$ for $r \in\left(r_{0}, \sqrt{2} / 2\right)$.

Proof. From (21) we clearly see that $h(r)$ can be rewritten as

$$
\begin{aligned}
h(r)= & \frac{\mathscr{E}(r)-r^{\prime 2} \mathscr{K}(r)}{r^{2}}\left[r^{\prime 2 / 3} \mathscr{K}(r)\right]^{-3} \\
& -\frac{16 p(1-p)}{\pi^{2}} .
\end{aligned}
$$

It follows from Lemma 2(1) and (2) together with (22) that $h(r)$ is strictly increasing on $(0, \sqrt{2} / 2)$.
Numerical computations show that

$$
h\left(0^{+}\right)=\frac{2\left[\pi^{2}-3 \mathscr{K}^{2}(\sqrt{2} / 2)\right]}{\pi^{2} \mathscr{K}^{2}(\sqrt{2} / 2)}=-0.02612 \ldots
$$

$<0$,

$$
\begin{aligned}
h\left(\frac{\sqrt{2}^{-}}{2}\right) & =\frac{4\left[\pi^{2} \mathscr{E}(\sqrt{2} / 2)-2 \mathscr{K}^{3}(\sqrt{2} / 2)\right]}{\pi^{2} \mathscr{K}^{3}(\sqrt{2} / 2)} \\
& =0.03708 \ldots>0 .
\end{aligned}
$$

Therefore, Lemma 3 follows easily from (23) and the monotonicity of $h(r)$ on the interval $(0, \sqrt{2} / 2)$.

\section{Main Results}

Theorem 4. The double inequality

$$
\begin{aligned}
Q^{\alpha_{1}}(a, b) A^{1-\alpha_{1}}(a, b) & <A G[A(a, b), Q(a, b)] \\
& <Q^{\beta_{1}}(a, b) A^{1-\beta_{1}}(a, b)
\end{aligned}
$$

holds for all $a, b>0$ with $a \neq b$ if and only if $\alpha_{1} \leq 1 / 2$ and $\beta_{1} \geq 2[\log \pi-\log \mathscr{K}(\sqrt{2} / 2)] / \log 2-1=0.5215 \ldots$

Proof. Since $A(a, b), Q(a, b)$, and $A G(a, b)$ are symmetric and homogenous of degree 1 , without loss of generality, we assume that $a>b>0$. Let $r=(a-b) / \sqrt{2\left(a^{2}+b^{2}\right)} \in(0, \sqrt{2} / 2)$. Then (2) and (3) lead to

$$
\begin{aligned}
& A G[A(a, b), Q(a, b)]=\frac{\pi A(a, b)}{2 r^{\prime} \mathscr{K}(r)}, \\
& Q(a, b)=\frac{A(a, b)}{r^{\prime}}, \\
& \frac{\log A G[A(a, b), Q(a, b)]-\log A(a, b)}{\log Q(a, b)-\log A(a, b)} \\
& =\frac{\log \mathscr{K}(r)+\log r^{\prime}+\log 2-\log \pi}{\log r^{\prime}} .
\end{aligned}
$$

Let

$$
\begin{aligned}
& f_{1}(r)=\log \mathscr{K}(r)+\log r^{\prime}+\log 2-\log \pi, \\
& f_{2}(r)=\log r^{\prime} \\
& f(r)=\frac{f_{1}(r)}{f_{2}(r)} .
\end{aligned}
$$

Then simple computations give

$$
\begin{aligned}
f_{1}\left(0^{+}\right) & =f_{2}\left(0^{+}\right)=0, \\
\frac{f_{1}^{\prime}(r)}{f_{2}^{\prime}(r)} & =\frac{\mathscr{K}(r)-\mathscr{E}(r)}{r^{2} \mathscr{K}(r)} .
\end{aligned}
$$

It follows from Lemmas 1, 2(3) and (27) and (28) that

$$
f\left(0^{+}\right)=\frac{1}{2}
$$

and $f(r)$ is strictly increasing on the interval $(0, \sqrt{2} / 2)$. 
Note that

$$
f\left(\frac{\sqrt{2}^{-}}{2}\right)=\frac{2[\log \pi-\log \mathscr{K}(\sqrt{2} / 2)]}{\log 2}-1 .
$$

Therefore, Theorem 4 follows easily from (26), (27), (29), and (30) and the monotonicity of $f(r)$ on the interval $(0, \sqrt{2} / 2)$

Remark 5. The left side inequality of Theorem 4 for $\alpha_{1} \leq 1 / 2$ can be derived directly from the fact that $A G(a, b)>G(a, b)=$ $\sqrt{a b}$ and $Q(a, b)>A(a, b)$ for all $a, b>0$ with $a \neq b$.

Theorem 6. The double inequality

$$
\begin{gathered}
\alpha_{2} Q(a, b)+\left(1-\alpha_{2}\right) A(a, b)<A G[A(a, b), Q(a, b)] \\
<\beta_{2} Q(a, b)+\left(1-\beta_{2}\right) A(a, b)
\end{gathered}
$$

holds for all $a, b>0$ with $a \neq b$ if and only if $\alpha_{2} \leq[\pi-$ $\sqrt{2} \mathscr{K}(\sqrt{2} / 2)] /[(2-\sqrt{2}) \mathscr{K}(\sqrt{2} / 2)]=0.4783 \ldots$ and $\beta_{2} \geq 1 / 2$.

Proof. Without loss of generality, we assume that $a>b>0$. Let $r=(a-b) / \sqrt{2\left(a^{2}+b^{2}\right)} \in(0, \sqrt{2} / 2)$. Then it follows from (2) and (3) that

$$
\frac{A G[A(a, b), Q(a, b)]-A(a, b)}{Q(a, b)-A(a, b)}=\frac{\pi / 2 \mathscr{K}(r)-r^{\prime}}{1-r^{\prime}} .
$$

Let

$$
\begin{aligned}
& g_{1}(r)=\frac{\pi}{2 \mathscr{K}(r)}-r^{\prime}, \\
& g_{2}(r)=1-r^{\prime}, \\
& g(r)=\frac{g_{1}(r)}{g_{2}(r)} .
\end{aligned}
$$

Then simple computations lead to

$$
\begin{aligned}
& g_{1}\left(0^{+}\right)=g_{2}\left(0^{+}\right)=0, \\
& \frac{g_{1}^{\prime}(r)}{g_{2}^{\prime}(r)}=1-\frac{\pi}{2} \frac{\mathscr{E}(r)-r^{\prime 2} \mathscr{K}(r)}{r^{2}}\left[r^{1 / 2} \mathscr{K}(r)\right]^{-2} .
\end{aligned}
$$

It follows from Lemmas 1, 2(1) and (2) together with (33) and (34) that

$$
g\left(0^{+}\right)=\frac{1}{2}
$$

and $g(r)$ is strictly decreasing on the interval $(0, \sqrt{2} / 2)$.

Note that

$$
g\left(\frac{\sqrt{2}^{-}}{2}\right)=\frac{\pi-\sqrt{2} \mathscr{K}(\sqrt{2} / 2)}{(2-\sqrt{2}) \mathscr{K}(\sqrt{2} / 2)} .
$$

Therefore, Theorem 6 follows easily from (32), (33), (35), and (36) and the monotonicity of $g(r)$ on the interval $(0, \sqrt{2} / 2)$
Remark 7. The right side inequality of Theorem 6 for $\beta_{2} \geq 1 / 2$ can be derived directly from the fact that $A G(a, b)<A(a, b)=$ $(a+b) / 2$ and $Q(a, b)>A(a, b)$ for all $a, b>0$ with $a \neq b$.

Theorem 8. Let $\alpha_{3}, \beta_{3} \in(1 / 2,1)$. Then the double inequality

$$
\begin{aligned}
Q & {\left[\alpha_{3} a+\left(1-\alpha_{3}\right) b, \alpha_{3} b+\left(1-\alpha_{3}\right) a\right] } \\
& <A G[A(a, b), Q(a, b)] \\
& <Q\left[\beta_{3} a+\left(1-\beta_{3}\right) b, \beta_{3} b+\left(1-\beta_{3}\right) a\right]
\end{aligned}
$$

holds for all $a, b>0$ with $a \neq b$ if and only if $\alpha_{3} \leq 1 / 2+$ $\sqrt{2} \sqrt{\pi^{2}-2 \mathscr{K}^{2}(\sqrt{2} / 2)} /[4 \mathscr{K}(\sqrt{2} / 2)]=0.8299 \ldots$ and $\beta_{3} \geq$ $1 / 2+\sqrt{2} / 4=0.8535 \ldots$

Proof. Without loss of generality, we assume that $a>b>0$. Let $r=(a-b) / \sqrt{2\left(a^{2}+b^{2}\right)} \in(0, \sqrt{2} / 2)$ and $p \in(1 / 2,1)$. Then (2) and (3) lead to

$$
\begin{aligned}
& Q[p a+(1-p) b, p b+(1-p) a] \\
& =\frac{\sqrt{1-4 p(1-p) r^{2}}}{r^{\prime}} A(a, b), \\
& Q[p a+(1-p) b, p b+(1-p) a] \\
& -A G[A(a, b), Q(a, b)] \\
& =\frac{A(a, b)}{\left[\sqrt{1-4 p(1-p) r^{2}}+\pi /[2 \mathscr{K}(r)]\right] r^{\prime}} H(r),
\end{aligned}
$$

where

$$
\begin{aligned}
H(r) & =1-4 p(1-p) r^{2}-\frac{\pi^{2}}{4 \mathscr{K}^{2}(r)}, \\
H\left(0^{+}\right) & =0 \\
H\left(\frac{\sqrt{2}^{-}}{2}\right) & =1-2 p(1-p)-\frac{\pi^{2}}{4 \mathscr{K}^{2}(\sqrt{2} / 2)}, \\
H^{\prime}(r) & =\frac{\pi^{2} r}{2} h(r),
\end{aligned}
$$

where $h(r)$ is defined by (21).

We divide the proof into four cases.

Case $1\left(p=p_{0}=1 / 2+\sqrt{2} \sqrt{\pi^{2}-2 \mathscr{K}^{2}(\sqrt{2} / 2)} /[4 \mathscr{K}(\sqrt{2} / 2)]\right)$. Then (42) becomes

$$
H\left({\frac{\sqrt{2}^{-}}{2}}^{-}\right)=0
$$

It follows from Lemma 3 and (43) that there exists $r_{0} \epsilon$ $(0, \sqrt{2} / 2)$ such that $H(r)$ is strictly decreasing on $\left(0, r_{0}\right]$ and strictly increasing on $\left[r_{0}, \sqrt{2} / 2\right)$. Therefore,

$$
\begin{gathered}
Q\left[p_{0} a+\left(1-p_{0}\right) b, p_{0} b+\left(1-p_{0}\right) a\right] \\
<A G[A(a, b), Q(a, b)]
\end{gathered}
$$


follows from (39), (41), and (44) together with the piecewise monotonicity of $H(r)$ on the interval $(0, \sqrt{2} / 2)$.

Case $2\left(p=p_{0}^{*}=1 / 2+\sqrt{2} / 4\right)$. Then we clearly see that

$$
\begin{aligned}
Q & {\left[p_{0}^{*} a+\left(1-p_{0}^{*}\right) b, p_{0}^{*} b+\left(1-p_{0}^{*}\right) a\right] } \\
& =\sqrt{\frac{A^{2}(a, b)+Q^{2}(a, b)}{2}}=Q[A(a, b), Q(a, b)] \\
& >A[A(a, b), Q(a, b)]>A G[A(a, b), Q(a, b)] .
\end{aligned}
$$

Case $3\left(1 / 2+\sqrt{2} \sqrt{\pi^{2}-2 \mathscr{K}^{2}(\sqrt{2} / 2)} /[4 \mathscr{K}(\sqrt{2} / 2)]<p<1\right)$. Then (42) leads to

$$
H\left(\frac{\sqrt{2}}{2}^{-}\right)>0
$$

Equation (39) and inequality (47) imply that there exists small enough $0<\delta_{1}<\sqrt{2} / 2$ such that

$$
\begin{gathered}
Q[p a+(1-p) b, p b+(1-p) a] \\
>A G[A(a, b), Q(a, b)]
\end{gathered}
$$

for all $a, b>0$ with $|a-b| / \sqrt{2\left(a^{2}+b^{2}\right)} \in\left(\sqrt{2} / 2-\delta_{1}, \sqrt{2} / 2\right)$. Case $4(1 / 2<p<1 / 2+\sqrt{2} / 4)$. Then (40) leads to

$$
H(r)=\left[4\left(p-\frac{1}{2}\right)^{2}-\frac{1}{2}\right] r^{2}+o\left(r^{2}\right) .
$$

Note that

$$
\left[4\left(p-\frac{1}{2}\right)^{2}-\frac{1}{2}\right] r^{2}<0
$$

Equations (39) and (49) together with inequality (50) imply that there exists small enough $0<\delta_{2}<\sqrt{2} / 2$ such that

$$
\begin{gathered}
Q[p a+(1-p) b, p b+(1-p) a] \\
<A G[A(a, b), Q(a, b)]
\end{gathered}
$$

for all $a, b>0$ with $|a-b| / \sqrt{2\left(a^{2}+b^{2}\right)} \in\left(0, \delta_{2}\right)$.

\section{Applications}

In this section, we use Theorems 4,6 , and 8 to present several bounds for the complete elliptic integrals $\mathscr{K}(r)$ and $\mathscr{E}(r)$.

From Theorems 4, 6, and 8 we get Theorem 9 immediately.

Theorem 9. Let $\lambda_{1}=2[\log \pi-\log \mathscr{K}(\sqrt{2} / 2)] / \log 2-1=$ $0.5215 \ldots, \lambda_{2}=[\pi-\sqrt{2} \mathscr{K}(\sqrt{2} / 2)] /[(2-\sqrt{2}) \mathscr{K}(\sqrt{2} / 2)]=$
$0.4783 \ldots$, and $\lambda_{3}=2-\pi^{2} /\left[2 \mathscr{K}^{2}(\sqrt{2} / 2)\right]=0.5644 \ldots$. Then the double inequalities

$$
\begin{aligned}
\frac{\pi}{2\left(1-r^{2}\right)^{\left(1-\lambda_{1}\right) / 2}} & <\mathscr{K}(r) \\
& <\frac{\pi}{2\left[\lambda_{2}+\left(1-\lambda_{2}\right) \sqrt{1-r^{2}}\right]}, \\
\frac{\pi}{2 \sqrt{1-(1 / 2) r^{2}}} & <\mathscr{K}(r)<\frac{\pi}{2 \sqrt{1-\lambda_{3} r^{2}}}
\end{aligned}
$$

hold for all $r \in(0, \sqrt{2} / 2)$.

It follows from the inequality

$$
\frac{\pi^{2}}{4}<\mathscr{E}(r) \mathscr{K}(r)<\frac{\pi^{2}}{4 \sqrt{r^{\prime}}}
$$

given in [24] that

$$
\frac{\pi^{2}}{4 \mathscr{K}(r)}<\mathscr{E}(r)<\frac{\pi^{2}}{4 \sqrt{r^{\prime}} \mathscr{K}(r)} .
$$

Theorem 9 and (54) lead to the following.

Theorem 10. Let $\lambda_{1}=2[\log \pi-\log \mathscr{K}(\sqrt{2} / 2)] / \log 2-1=$ $0.5215 \ldots, \lambda_{2}=[\pi-\sqrt{2} \mathscr{K}(\sqrt{2} / 2)] /[(2-\sqrt{2}) \mathscr{K}(\sqrt{2} / 2)]=$ $0.4783 \ldots$, and $\lambda_{3}=2-\pi^{2} /\left[2 \mathscr{K}^{2}(\sqrt{2} / 2)\right]=0.5644 \ldots$ Then the double inequalities

$$
\begin{aligned}
\frac{\pi}{2}\left[\lambda_{2}+\left(1-\lambda_{2}\right) r^{\prime}\right] & <\mathscr{E}(r)<\frac{\pi}{2} r^{\prime(1 / 2)-\lambda_{1}}, \\
\frac{\pi}{2} \sqrt{1-\lambda_{3} r^{2}} & <\mathscr{E}(r)<\frac{\pi}{2} \sqrt{\frac{2-r^{2}}{2 r^{\prime}}}
\end{aligned}
$$

hold for all $r \in(0, \sqrt{2} / 2)$.

\section{Competing Interests}

The authors declare that there is no conflict of interests regarding the publication of this paper.

\section{Acknowledgments}

The research was supported by the Natural Science Foundation of China under Grants 11371125, 61374086, and 11401191, the Natural Science Foundation of Zhejiang Province under Grant LY13A010004, and the Natural Science Foundation of the Zhejiang Broadcast and TV University under Grant XKT$15 \mathrm{G} 17$.

\section{References}

[1] J. M. Borwein and P. B. Borwein, "The arithmetic-geometric mean and fast computation of elementary functions," SIAM Review, vol. 26, no. 3, pp. 351-366, 1984.

[2] J. M. Borwein and P. B. Borwein, Pi and the AGM, John Wiley \& Sons, New York, NY, USA, 1987. 
[3] G. Almkvist and B. Berndt, "Gauss, landen, ramanujan, the arithmetic-geometric mean, ellipses, $\pi$ and the ladies diary," The American Mathematical Monthly, vol. 95, no. 7, pp. 585-608, 1988.

[4] B. C. Carlson, "Hidden symmetries of special functions," SIAM Review, vol. 12, pp. 332-345, 1970.

[5] I. Elishakoff, V. Birman, and J. Singer, "Influence of initial imperfections on nonlinear free vibration of elastic bars," Acta Mechanica, vol. 55, no. 3-4, pp. 191-202, 1985.

[6] D. K. Lee, "Application of theta functions for numerical evaluation of complete elliptic integrals of the first and second kinds," Computer Physics Communications, vol. 60, no. 3, pp. 319-327, 1990.

[7] T. Horiguchi, "Lattice Green's function for anisotropic triangular lattice," Physica A, vol. 178, no. 2, pp. 351-363, 1991.

[8] K. Mayrhofer and F. D. Fischer, "Derivation of a new analytical solution for a general two-dimensional finite-part integral applicable in fracture mechanics," International Journal for Numerical Methods in Engineering, vol. 33, no. 5, pp. 1027-1047, 1992.

[9] C. C. Maican, Integral Evaluations Using the Gamma and Beta Functions and Elliptic Integrals in Engineering, International Press, Cambridge, Mass, USA, 2005.

[10] J. M. Borwein and P. B. Borwein, "Inequalities for compound mean iterations with logarithmic asymptotes," Journal of Mathematical Analysis and Applications, vol. 177, no. 2, pp. 572-582, 1993.

[11] P. Bracken, "An arithmetic-geometric mean inequality," Expositiones Mathematicae, vol. 19, no. 3, pp. 273-279, 2001.

[12] Zh.-H. Yang, "A new proof of inequalities for Gauss compound mean," International Journal of Mathematical Analysis, vol. 4, no. 21-24, pp. 1013-1018, 2010.

[13] B. C. Carlson and M. Vuorinen, "Problem 91-17," SIAM Review, vol. 33 , no. 4 , p. $655,1991$.

[14] M. K. Vamanamurthy and M. Vuorinen, "Inequalities for means," Journal of Mathematical Analysis and Applications, vol. 183, no. 1, pp. 155-166, 1994.

[15] J. Sándor, "On certain inequalities for means," Journal of Mathematical Analysis and Applications, vol. 189, no. 2, pp. 602606, 1995.

[16] J. Sándor, “On certain inequalities for means II," Journal of Mathematical Analysis and Applications, vol. 199, no. 2, pp. 629635, 1996.

[17] E. Neuman and J. Sándor, "On the Schwab-Borchardt mean," Mathematica Pannonica, vol. 14, no. 2, pp. 253-266, 2003.

[18] R. Kühnau, "Eine Methode, die Positivität einer Funktion zu prüfen," Zeitschrift für Angewandte Mathematik und Mechanik, vol. 74, no. 2, pp. 140-143, 1994.

[19] S.-L. Qiu and M. K. Vamanamurthy, "Sharp estimates for complete elliptic integrals," SIAM Journal on Mathematical Analysis, vol. 27, no. 3, pp. 823-834, 1996.

[20] H. Alzer and S.-L. Qiu, "Monotonicity theorems and inequalities for the complete elliptic integrals," Journal of Computational and Applied Mathematics, vol. 172, no. 2, pp. 289-312, 2004.

[21] Y.-M. Chu and M.-K. Wang, "Inequalities between arithmeticgeometric, Gini, and Toader means," Abstract and Applied Analysis, vol. 2012, Article ID 830585, 11 pages, 2012.

[22] Z.-H. Yang, Y.-Q. Song, and Y.-M. Chu, "Sharp bounds for the arithmetic-geometric mean," Journal of Inequalities and Applications, vol. 2014, article 192, 2014.
[23] Zh.-H. Yang, Y.-M. Chu, and W. Zhang, "Accurate approximations for the complete elliptic integral of the second kind," Journal of Mathematical Analysis and Applications, vol. 438, no. 2, pp. 875-888, 2016.

[24] G. D. Anderson, M. K. Vamanamurthy, and M. Vuorinen, Conformal Invariants, Inequalities, and Quasiconformal Maps, John Wiley \& Sons, New York, NY, USA, 1997. 


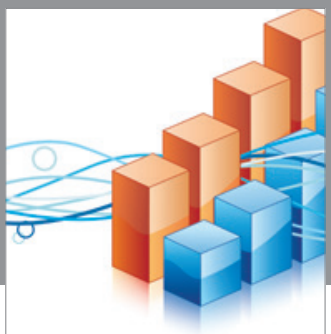

Advances in

Operations Research

vatem alat4

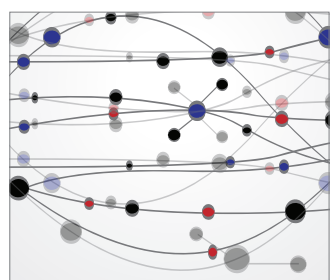

\section{The Scientific} World Journal
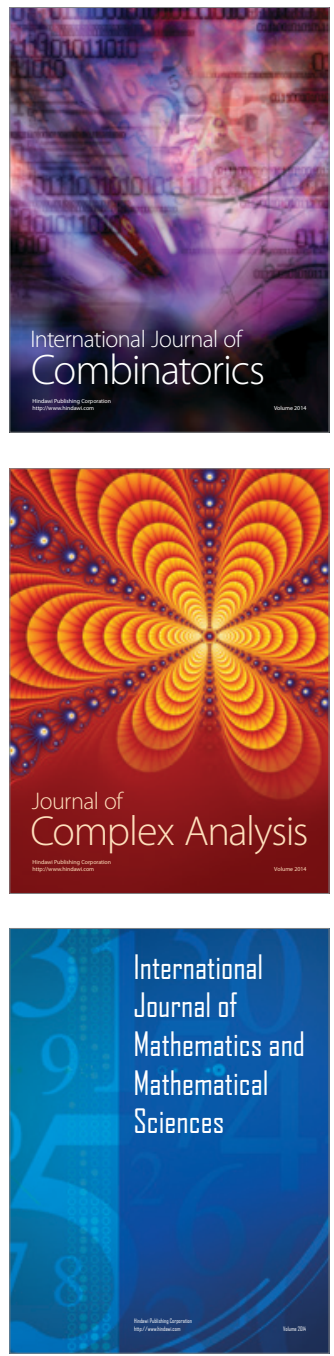
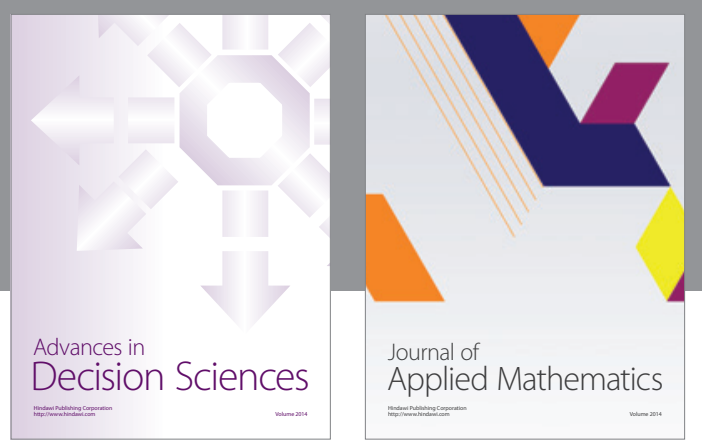

Algebra

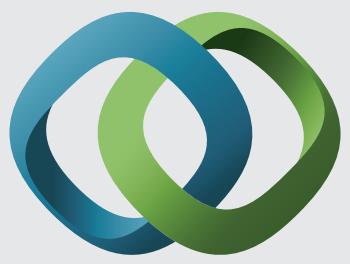

\section{Hindawi}

Submit your manuscripts at

http://www.hindawi.com
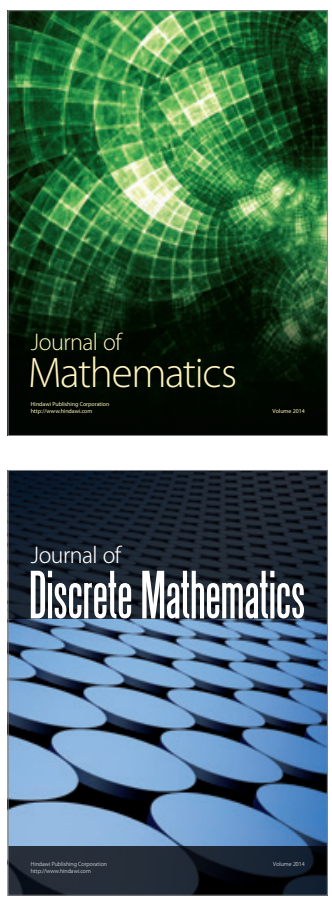

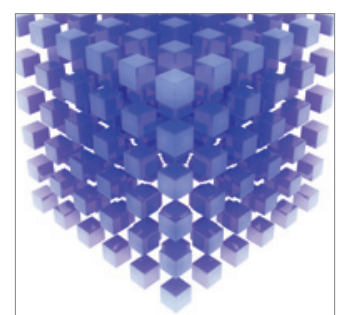

Mathematical Problems in Engineering
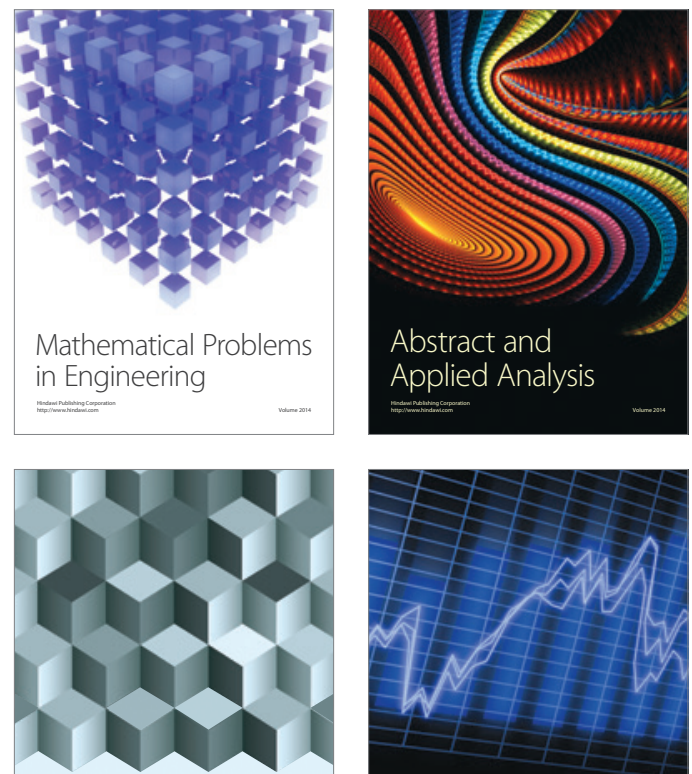

Journal of

Function Spaces

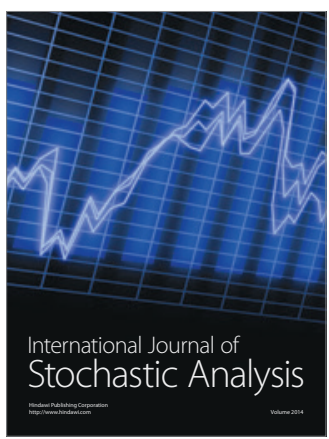

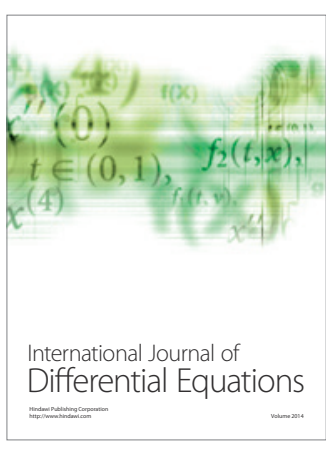
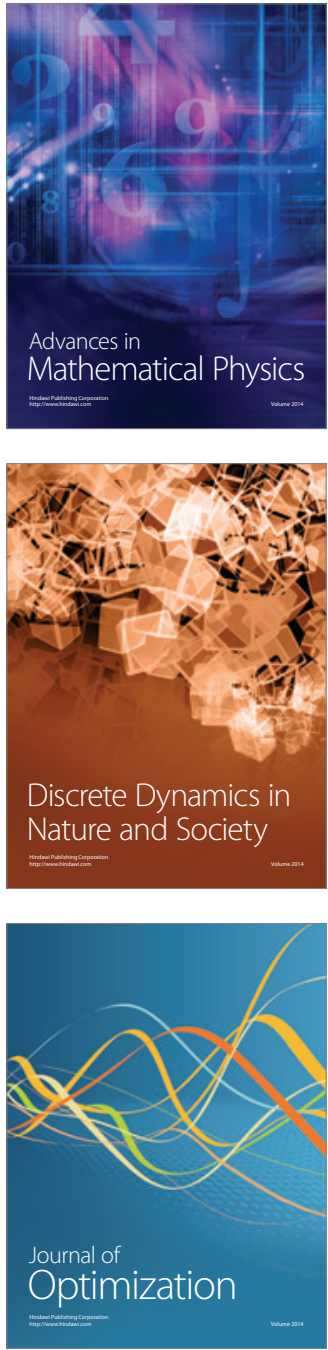